\title{
Estrategias para el fortalecimiento de la cultura organizacional de la Agencia Nacional de Hidrocarburos*
}

[Artículos de investigación]

\author{
Ingrid Astrid Ochoa Vivas ${ }^{* *}$ \\ Ángela Mónica Castro Mora ${ }^{* * *}$
}

Recibido: o9 de marzo de 2020

Revisado: 12 de agosto de 2020

Aceptado: 1 de septiembre de 2020

Cómo citar este artículo:

Ochoa Vivas, I. A. y Castro Mora, A. M. (2020). Estrategias para el fortalecimiento de la cultura organizacional de la Agencia Nacional de Hidrocarburos. Signos, Investigación en sistemas de gestión, 13(1).

https://doi.org/10.15332/24631140.6347

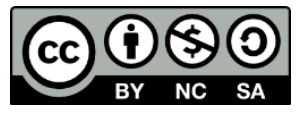

\footnotetext{
* Artículo resultado de investigación

** Universidad Santo Tomás. Especialista en Administración y Gerencia de Sistemas de Calidad. Ingeniera industrial. Bogotá, Colombia. Correo electrónico:

ingridvivas22@hotmail.com. ORCID: https://orcid.org/0000-0002-6127-3083. CvLAC: https://scienti.minciencias.gov.co/cvlac/visualizador/generarCurriculoCv.do?cod_rh $=0$ 001831647

*** Universidad Santo Tomás. Especialista en Administración y Gerencia de Sistemas de Calidad. Administradora pública. Bogotá, Colombia. Correo electrónico: angecasm@hotmail.com. ORCID: https://orcid.org/0000-0001-8697-3257. CvLAC: https://scienti.minciencias.gov.co/cvlac/visualizador/generarCurriculoCv.do?cod_rh $=0$ $\underline{001831497}$
} 


\section{Resumen}

El objetivo de esta investigación es caracterizar la cultura organizacional de la Agencia Nacional de Hidrocarburos mediante la metodología propuesta por Cameron y Quinn (1999), que se basa en el modelo de valores por competencia. Con un enfoque mixto, se recopilaron datos de diferentes dimensiones de la cultura organizacional a través del cuestionario Organizational Cultural Assessment Instrument (OCAI), diseñado y validado estadísticamente por estos autores. La población de estudio estuvo conformada por un total de 220 colaboradores de todos los niveles de la Agencia Nacional de Hidrocarburos. A partir de los datos obtenidos, se determinó la cultura actual, la cultura deseada y la cultura requerida con base en cuatro clases de culturas genéricas: clan/colaboración, jerárquica/control, adhocracia/creación y mercado/competitividad. Las diferencias y similitudes encontradas entre los tipos de cultura evidenciaron las brechas respecto a la cultura requerida y la cultura deseada.

Palabras clave: cultura organizacional, marco de valores en competencia, sistemas de gestión.

\section{Strategies for strengthening the organizational culture of the National Hydrocarbons Agency.}

\section{Abstract}

This research aims at characterizing the organizational culture of the National Hydrocarbons Agency through the methodology proposed by Cameron and Quinn (1999), which is based on the values by competence model. Using a mixed approach, data from different dimensions of the organizational culture were collected through the Organizational Cultural Assessment Instrument (OCAI) questionnaire, designed, and statistically validated by these authors. The study population was made up of a total of 220 collaborators from all National Hydrocarbons Agency instances. From the data obtained, the current culture, the desired culture and the required culture were determined based on four classes of generic 
cultures: clan/collaboration, hierarchical/control, adhocracy/creation, and market/competitiveness. The differences and similarities detected between the types of culture stressed the gaps between the required and the desired culture.

Keywords: organizational culture, framework of competing values, management systems.

\section{Estratégias para o fortalecimento da cultura organizacional da Agência Nacional de Hidrocarbonetos}

\section{Resumo}

O objetivo desta pesquisa é caracterizar a cultura organizacional da Agência Nacional de Hidrocarbonetos através da metodologia proposta por Cameron e Quinn (1999), baseada nos valores por modelo de competência. Usando uma abordagem mista, os dados de diferentes dimensões da cultura organizacional foram coletados através do questionário Instrumento de Avaliação da Cultura Organizacional (OCAI, do inglês Organizational Cultural Assessment Instrument), projetado e validado estatisticamente por esses autores. A população do estudo era composta por um total de 220 colaboradores de todos os níveis da Agência Nacional de Hidrocarbonetos. A partir dos dados obtidos, a cultura atual, a cultura desejada e a cultura necessária foram determinadas a partir de quatro classes de culturas genéricas: clã/colaboração, hierárquica/controle, adhocracia/criação e mercado/concorrência. As diferenças e semelhanças encontradas entre os tipos de cultura destacaram as lacunas entre a cultura necessária e a cultura desejada.

Palavras-chave: cultura organizacional, quadro de valores em concorrência, sistemas de gestão. 


\section{Introducción}

La cultura organizacional es un recurso o activo organizacional que, al ser gestionado correctamente desde sus valores, símbolos, lenguaje, ideología, creencias y ritos, puede afectar el rendimiento de los integrantes de las empresas y, de esta forma, facilitar la implementación y mejora de los sistemas de gestión. Los sistemas de gestión son procesos, acciones y tareas que se llevan a cabo sobre un conjunto de elementos para lograr el éxito sostenido de una organización; es decir, disponer de capacidad para satisfacer las necesidades y las expectativas de sus clientes, y mejorar constantemente en diferentes aspectos de su gestión. Es importante resaltar que la cultura organizacional es un potencial activo estratégico desde la perspectiva de la administración, y las empresas con una cultura original giran en torno a valores claves según los requerimientos de sus clientes, lo que agrega valor a sus acciones, maximiza los resultados y las consolida (Rodríguez, 2009).

La cultura organizacional es una estrategia que permite desarrollar ventajas competitivas y un buen servicio, que es lo que los ciudadanos esperan de las entidades públicas y lo que se pretende lograr con la implementación de los sistemas de gestión. Esta propuesta de investigación presenta las estrategias para el fortalecimiento de la cultura organizacional en la Agencia Nacional de Hidrocarburos (ANH) para facilitar la implementación y mejora de los sistemas de gestión que se adoptaron al interior, con el fin de contribuir al mejoramiento de la gestión y el logro de los objetivos institucionales.

La investigación permitió identificar que, en las organizaciones públicas, la cultura organizacional es un concepto con poca importancia, pues se desconoce su peso e influencia en los procesos de cambio y éxito organizacional. Generalmente, se establece una cultura organizacional que se caracteriza por una rigidez muy marcada en los procesos, falta de 
enfoque hacia la gestión estratégica del talento humano, carencia de métodos de estimulación hacia el empleado, falta de incentivos por el nivel de productividad, entre otros. Es decir, se observan elementos característicos de una cultura que no favorece los procesos de cambio y mejora organizacional.

Para dar respuesta al problema, se plantearon tres momentos en la investigación. Primero, se identificó la cultura organizacional en la ANH a través de encuestas utilizando el modelo de Cameron y Quinn (1999), basado en el modelo de valores en competencia. Luego, se identificó la brecha entre la cultura actual y la cultura organizacional deseada, orientada a la implementación y mejora de los sistemas de gestión. Finalmente, se establecieron estrategias para el cierre de brechas entre la cultura organizacional requerida y la actual que facilitan la implementación y mejora de los sistemas de gestión (Barahona y Rodríguez, 2015).

La propuesta investigativa permite identificar los elementos de la cultura organizacional que se convierten en factores claves de éxito en la ANH para implementar procesos de cambio con el propósito de establecer las estrategias para crear una estructura más eficiente.

\section{Metodología}

El objetivo de esta investigación fue caracterizar la cultura organizacional de la ahn mediante la metodología propuesta por Cameron y Quinn (1999), basada en el modelo de valores por competencia. La población objeto del estudio estuvo conformada por un total de 220 colaboradores de la ahn y la muestra analizada incluyó todos los niveles de la entidad. 


\section{Instrumento de medición}

El cuestionario elaborado por Cameron y Quinn (1999) permite determinar la cultura actual de la organización, así como la cultura deseada por los integrantes de la empresa. Se aplicó en organizaciones de diferentes sectores económicos para diagnosticar la cultura organizacional de forma exitosa.

El cuestionario contó con seis preguntas cerradas, cada una de las cuales tenía cuatro opciones de respuesta señaladas con las letras A, B, C y D. Cada opción está asociada con uno de los cuatro tipos de cultura señalados por Cameron y Quinn. El participante deberá asignar una puntuación a cada opción de respuesta para obtener un total de 100 puntos. El puntaje mayor se otorgó a la opción de respuesta que describía mejor a la empresa. La opción A mide el grado de orientación de la empresa a la cultura de clan; la opción B mide el grado de orientación de la empresa a la cultura adhocracia; la alternativa $\mathrm{C}$ mide el grado de orientación de la empresa a la cultura de mercado, y la opción D mide el grado de orientación de la empresa a la cultura jerarquizada. El resultado se obtiene de la sumatoria de todas las puntuaciones de una opción de respuesta y se divide entre 6; de esta manera se obtienen los resultados por letra.

\section{Diseño muestral}

Para determinar la cultura requerida se invitó a los líderes de los sistemas de gestión de cada proceso a responder el cuestionario diseñado y validado por Cameron y Quinn. En total, se trabajó con una población de 15 personas. La población o universo es el "conjunto de todos los casos que concuerdan con determinadas especificaciones" (Hernandéz-Sampieri et ál., 2010, p. 174). Por otro lado, para identificar la cultura actual de la ANH se utilizó una muestra probabilística estratificada. Se invitó a responder el cuestionario a todos los colaboradores (220 personas) de la 
ANH; sin embargo, se obtuvo respuesta de al menos 55 personas de las diferentes áreas de trabajo o niveles de responsabilidad.

La muestra probabilística es el subgrupo de la población en el que todos los elementos tienen la misma posibilidad de ser elegidos. La muestra probabilística estratificada es el "muestreo en el que la población se divide en segmentos y se selecciona una muestra para cada segmento" (Hernandéz-Sampieri et ál., 2010, p. 180). Para determinar el tamaño de una muestra representativa de todos los colaboradores de la $\mathrm{ANH}$, con cierta posibilidad de error, nivel de confianza y probabilidad, se establecieron los siguientes criterios: un total de 220 colaboradores, con un error máximo aceptable de 10 \% y un nivel de confianza de $90 \%$. Así, se obtuvo un tamaño de muestra de 52 personas. Este dato estará sujeto a modificación si el tamaño de la población varía. Es importante señalar que, a través del área a cargo de la gestión del recurso humano de la entidad, se facilitaron los mecanismos para enviar el cuestionario de Cameron y Quinn (1999).

\section{Recolección y análisis de datos}

Se debe tener en cuenta que "recolectar los datos implica elaborar un plan detallado de procedimientos que nos conduzcan a reunir datos con un propósito específico" (Hernandéz-Sampieri et ál., 2010, p. 198). La recolección de información se realizó en las siguientes etapas:

\section{Revisión y comprensión de información clave}

La revisión documental permitió identificar aspectos relevantes asociados a la cultura organizacional, tales como direccionamiento estratégico, mapa de procesos, medición de clima, organización y desempeño, informes de gestión, etc., que proveen un contexto de la realidad organizacional e 
impactan el despliegue de rasgos culturales que facilitan o dificultan la implementación y mejora de los sistemas de gestión.

\section{Aplicación del cuestionario para medir cultura actual}

Se invitó a todos los colaboradores de la ANH a responder el cuestionario para evaluar la cultura organizacional (OCAI) propuesto por Cameron y Quinn (1999). El procesamiento de datos se realizó una vez se obtuvo respuesta del total de colaboradores establecidos en el tamaño de la muestra.

Se comunicó a los colaboradores el objetivo del estudio, las etapas y cómo fue su participación a través de correo electrónico y piezas de comunicación. La aplicación de la encuesta se realizó a través de un tercero para el desarrollo de una aplicación como página web, con lenguaje de programación php para el servidor y typescript para la ANH. Los frameworks utilizados fueron Symfony para php y Angular para typescript, el cual estuvo habilitado por un periodo de tiempo que se acordó previamente con la entidad. Dicha aplicación permitió la recopilación de la información de forma rápida y manteniendo los niveles de confidencialidad de las respuestas de los colaboradores, según lo acordado con la entidad.

La encuesta se realizó en un periodo de cinco días en donde se realizaron 93 ingresos al aplicativo, de los cuales 31 funcionarios no dieron respuesta a la encuesta (solo ingresaron al aplicativo), 7 funcionarios dieron respuesta solo a una de las dimensiones (datos que no se tomaron para realizar el análisis de datos de esta investigación) y solo 55 encuestas se tomaron para realizar el análisis de la investigación, siendo este el $25 \%$ de la totalidad de los colaboradores de la ANH. 


\section{Aplicación del cuestionario para determinar la cultura requerida}

Se realizó un taller con los responsables de cada proceso que han trabajado en la implementación, mantenimiento y mejora de los sistemas de gestión, en el que se determinó la cultura requerida por la $\mathrm{ANH}$, con base en el cuestionario para evaluar la cultura organizacional (OCAI) propuesto por Cameron y Quinn (1999). Es importante señalar que la identificación de la cultura requerida debía responder a los retos establecidos en los lineamientos estratégicos de la empresa. El taller tuvo una duración estimada de dos horas, al final de las cuales se obtuvo un solo cuestionario respondido y aprobado por todo el equipo. A partir de este se diseñó la gráfica correspondiente de la cultura requerida en la $\mathrm{ANH}$.

\section{Análisis de datos y establecimiento de estrategias}

El procesamiento de datos se realizó a través de una aplicación como página web, con lenguaje de programación php para el servidor y typescript para la ANH. Los frameworks utilizados fueron Symfony para php y Angular para typescript. Para analizar los datos, se utilizó la información recopilada en el cuestionario aplicado y se identificó la cultura actual, la brecha entre la cultura actual y la requerida. Una vez se analizó la información, se diseñaron estrategias para el cierre de dichas brechas, de manera que se facilitara la implementación y mejora de los sistemas de gestión. Para esto también se tuvo en cuenta la documentación revisada anteriormente.

\section{Resultados y discusión}

\section{Cultura organizacional actual}

Para la interpretación de los resultados, es importante considerar las características de la evolución de la cultura (figura 1). 
Figura 1. Evolución de la cultura en las organizaciones

\section{Fle xibilidad}

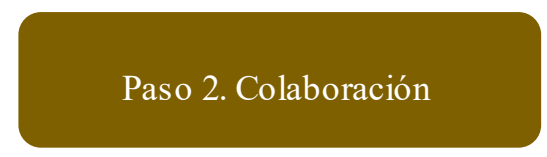

A medida que se desarrollan cambios, su estructura cambia hacia una de clan, un sentimiento familiar, un alto sentimiento de pertenencia y una identificación personal con la organización.

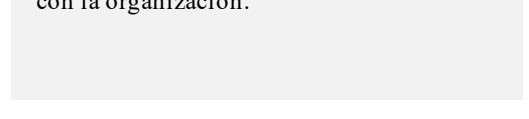

Interno

\section{Paso 3. Control}

Eventualmente se observa una crisis potencial. La organización se ve abocada a

la formalización de su estructura y la definición de procedimientos estandarizados para así controlar el incremento de la responsabilidad.

\section{4 \\ Paso 1. Creación}

En los primeros estados del ciclo de la vida, las organizaciones tienden a estar dominadas por este cuadrante. Adolecen de políticas formales y estructuras y son frecuentemente lideradas por un líder único, poderoso y visionario.

\section{Paso 4. Competitividad}

La organización jerarquizada es eventualmente reemplazada por una con enfoque en el cliente, la competencia, el logro de resultados y la construcción de relaciones externas.

\section{Control}

Fuente: Cameron y Quinn (1999).

Asimismo, se deben considerar los siguientes aspectos:

- Tipo: se refiere al perfil general de la ANH. Los cuadrantes en los que los puntajes son más altos indican los estilos organizacionales que 
tienden a ser más fuertes en la entidad. Identifica las asunciones básicas, los estilos y los valores que predominan.

- Discrepancias: se refiere a las discrepancias encontradas entre la cultura organizacional actual, la deseada y la requerida. Considera lo que debería cambiarse para poder alcanzar la cultura organizacional requerida.

- Fuerza: que una organización requiera de una cultura fuerte y dominante en oposición a un estilo ecléctico depende de las particularidades del entorno en el que esta se desempeña.

- Congruencia: todas las dimensiones de la cultura organizacional están alineadas: la estrategia, el estilo de liderazgo, el sistema de recompensas y la administración del recurso humano, entre otros. En organizaciones con incongruencia de estilos, los resultados sobre cada una de las dimensiones de la cultura enfatizarán en uno diferente, sin mostrar una semejanza o patrón particular.

\section{Resultados generales}

La identificación de brechas comienza con el análisis general de los datos obtenidos en los talleres realizados para identificar la cultura deseada y la requerida con colaboradores de la entidad expertos en la implementación y mejora de sistemas de gestión (tabla 1). En la figura 2 se ilustran las tres culturas que se identificaron con el cuestionario de Cameron y Quinn. 
Figura 2. Resultados de cultura general

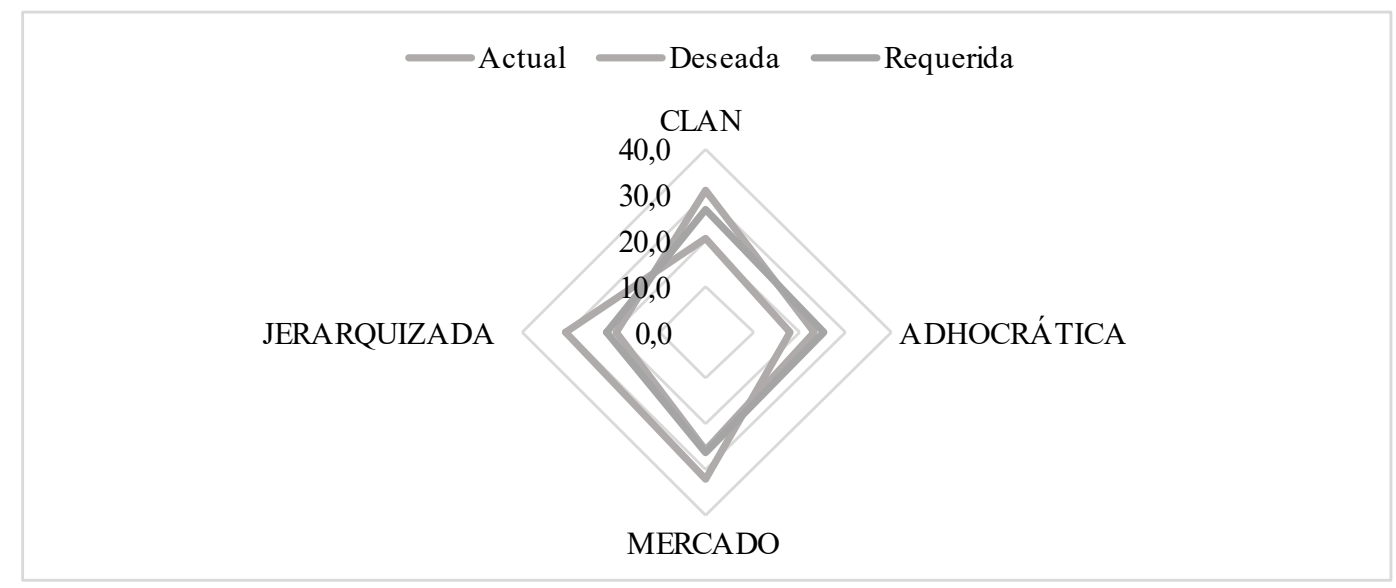

Fuente: elaboración propia.

Tabla 1. Resultado de cultura global con promedio por cada tipo

\begin{tabular}{|l|l|l|l|}
\multicolumn{1}{r|}{ Tipo de cultura } & \multicolumn{1}{c|}{ Actual } & \multicolumn{1}{c|}{ Deseada } & \multicolumn{1}{c|}{ Requerida } \\
\hline Clan & 20,3 & 31 & 26,5 \\
\hline Adhocrática & 18,3 & 23,5 & 25,5 \\
\hline Mercado & 32,1 & 25,7 & 26,5 \\
\hline Jerarquizada & 30,7 & 19,8 & 21,7 \\
\hline
\end{tabular}

Fuente: elaboración propia.

En la figura 2 se muestran las tres culturas de la ANH (actual, deseada y requerida) y se observa que la cultura actual es mercado, la cultura deseada es clan y la cultura requerida presenta un empate entre clan y mercado. La cultura clan, que corresponde a la identificada como deseada y requerida por la $\mathrm{ANH}$, es uno de los tipos de cultura que, según la literatura, favorece la implementación y mejora de los sistemas de gestión. Por lo tanto, se deben establecer estrategias para adoptar rasgos característicos de este tipo de cultura. 


\section{Caracterización organizacional}

En la dimensión de caracterización organizacional, la cultura actual es mercado, lo cual corresponde con el mismo tipo de cultura identificada en el resultado general. La cultura deseada es clan y la requerida es adhocrática. En esta dimensión, las culturas deseadas y las requeridas corresponden con los tipos de cultura que más favorecen la implementación de los sistemas de gestión. Teniendo en cuenta la brecha con la cultura actual, se requiere establecer estrategias para que la entidad sea más dinámica y emprendedora, y se perciba como una gran familia (figura 3, tabla 2).

Figura 3. Resultados de cultura en la dimensión de caracterización organizacional

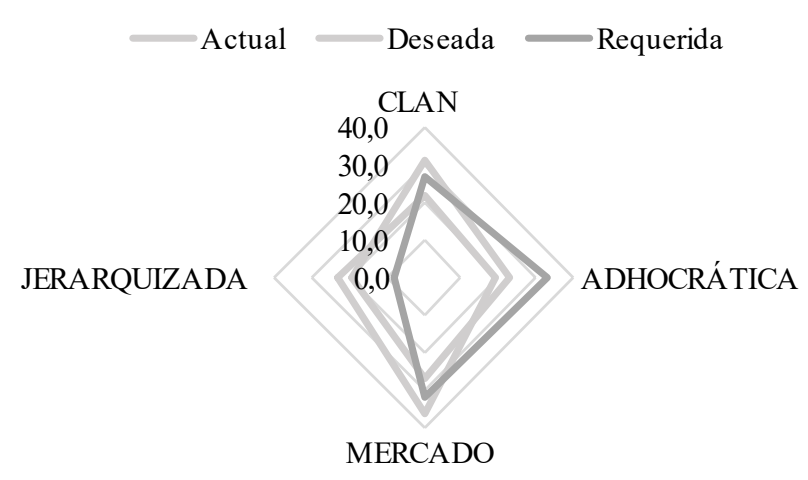

Fuente: elaboración propia.

Tabla 2. Resultados promedio en la dimensión de caracterización organizacional

\begin{tabular}{|l|l|l|l|}
\multicolumn{1}{|c|}{ Tipo de cultura } & \multicolumn{1}{c|}{ Actual } & \multicolumn{1}{c|}{ Deseada } & \multicolumn{1}{c}{ Requerida } \\
\hline Clan & 21,9 & 31 & 27 \\
\hline Adhocrática & 18,93 & 23 & 33 \\
\hline Mercado & 36,58 & 27 & 32 \\
\hline Jerarquizada & 23,1 & 19 & 8
\end{tabular}

Fuente: elaboración propia. 


\section{Dimensión liderazgo}

En la dimensión de liderazgo organizacional, la cultura actual es mercado, la deseada es clan y la requerida, adhocrática. En esta dimensión se presenta una gran brecha entre la cultura actual y las culturas deseada y requerida; por lo tanto, se requieren estrategias para que los líderes actúen como tutores, consejeros y buenos mentores (figura 4, tabla 3).

Figura 4. Resultado de cultura en la dimensión de liderazgo

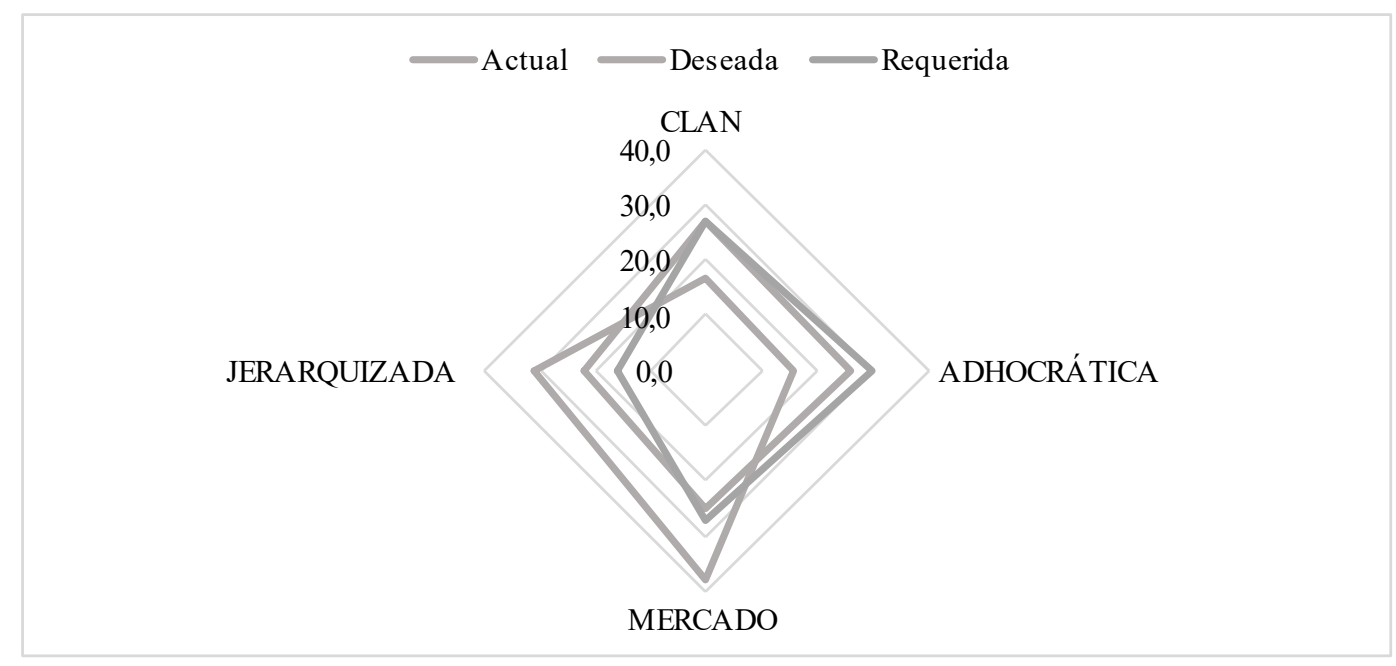

Fuente: elaboración propia.

Tabla 3. Resultados promedio de cultura en la dimensión de liderazgo

\begin{tabular}{|l|l|l|l|}
\multicolumn{1}{|c|}{ Tipo de cultura } & \multicolumn{1}{c|}{ Actual } & Deseada & Requerida \\
\hline Clan & 16,6 & 27 & 27 \\
\hline Adhocrática & 15,82 & 26 & 30 \\
\hline Mercado & 37,63 & 25 & 27 \\
\hline Jerarquizada & 31,28 & 22 & 16 \\
\hline
\end{tabular}

Fuente: elaboración propia. 


\section{Estilo de dirección}

En la dimensión de estilo de dirección, la cultura actual es mercado y la cultura deseada es clan, que corresponde con la requerida. Por lo tanto, se requiere reforzar el trabajo en equipo, el consenso y la participación (figura 5, tabla 4).

Figura 5. Resultado de cultura en la dimensión estilo de dirección

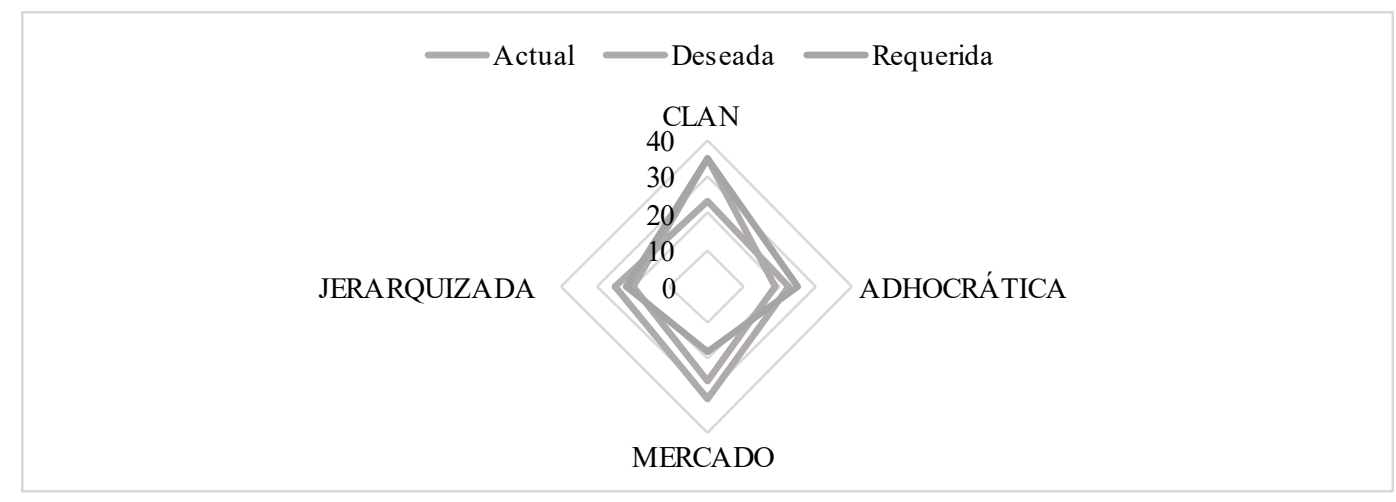

Fuente: elaboración propia.

Tabla 4. Resultados promedio de cultura en la dimensión estilo de dirección

\begin{tabular}{|l|l|l|l|}
\multicolumn{1}{|c|}{ Tipo de cultura } & \multicolumn{1}{c|}{ Actual } & Deseada & Requerida \\
\hline Clan & 23,23 & 35 & 35 \\
\hline Adhocrática & 22,36 & 19 & 25 \\
\hline Mercado & 30,77 & 26 & 18 \\
\hline Jerarquizada & 25,04 & 20 & 22 \\
\hline
\end{tabular}

Fuente: elaboración propia.

\section{Valores corporativos}

En la dimensión de valores compartidos, la cultura actual es jerarquizada y la deseada es mercado, que corresponde también con la requerida. En esta dimensión, la cultura deseada y la requerida identificadas no corresponden con un tipo de cultura que favorezca la implementación y mejora de los sistemas de gestión. Es importante implementar estrategias 
para adoptar rasgos característicos de la cultura clan o adhocrática, enfatizando en el compromiso con la innovación y el cambio (figura 6, tabla 5).

Figura 6. Resultados de cultura en la dimensión de valores corporativos

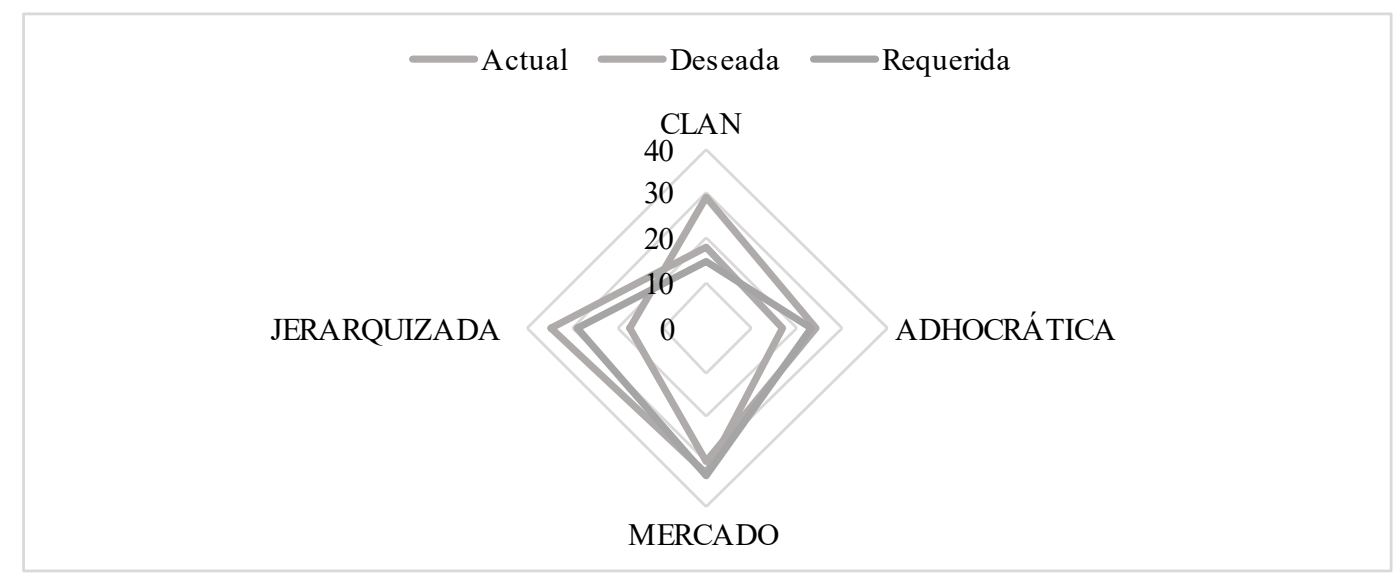

Fuente: elaboración propia.

Tabla 5. Resultados promedio de cultura en la dimensión de valores corporativos

\begin{tabular}{|l|l|l|l|}
\multicolumn{1}{|c|}{ Tipo de cultura } & \multicolumn{1}{c|}{ Actual } & Deseada & Requerida \\
\hline Clan & 17,82 & 29 & 15 \\
\hline Adhocrática & 16,59 & 24 & 23 \\
\hline Mercado & 32,6 & 30 & 33 \\
\hline Jerarquizada & 34,98 & 17 & 29 \\
\hline
\end{tabular}

Fuente: elaboración propia.

\section{Énfasis estratégico}

En la dimensión de énfasis estratégico, la cultura actual es jerarquizada, la deseada es clan y la requerida es adhocrática. Se requiere una entidad con mayor dinamismo, aceptación de nuevos retos, aprendizaje de los errores, mayor participación y mentalidad abierta (figura 7, tabla 6). 
Figura 7. Resultado de cultura en la dimensión de énfasis estratégico

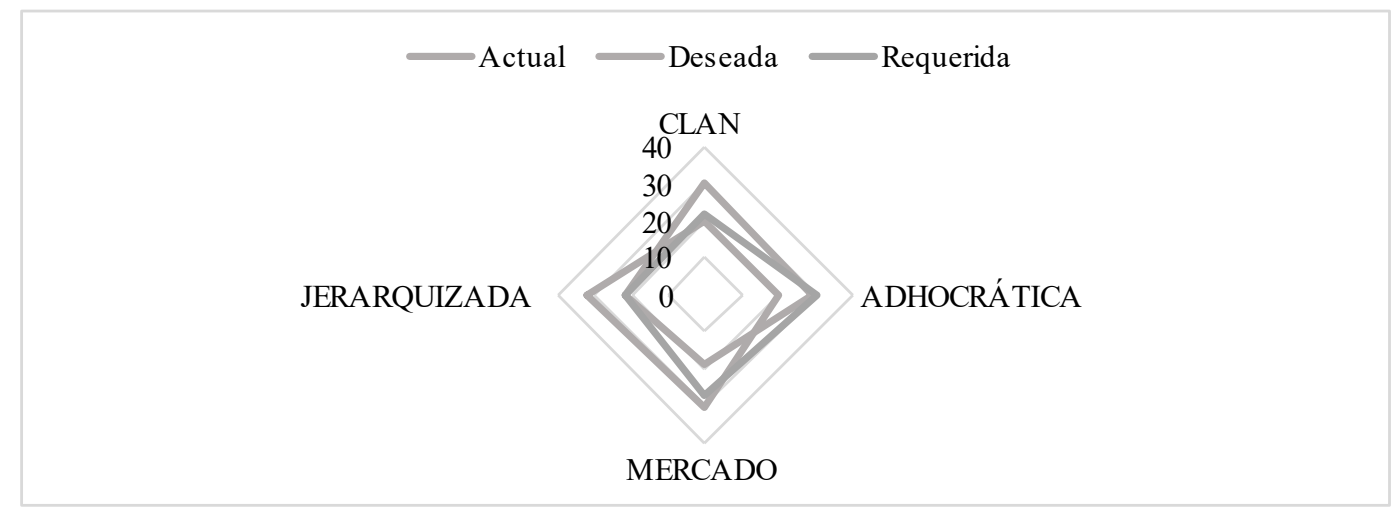

Fuente: elaboración propia.

Tabla 6. Resultados promedio de cultura en la dimensión de énfasis estratégico

\begin{tabular}{|l|l|l|l|}
\multicolumn{1}{|c|}{ Tipo de cultura } & \multicolumn{1}{c|}{ Actual } & \multicolumn{1}{c|}{ Deseada } & \multicolumn{1}{c|}{ Requerida } \\
\hline Clan & 19,65 & 30 & 22 \\
\hline Adhocrática & 19,98 & 29 & 30 \\
\hline Mercado & 30,51 & 19 & 27 \\
\hline Jerarquizada & 31,98 & 22 & 22 \\
\hline
\end{tabular}

Fuente: elaboración propia.

\section{Criterios de éxito}

En la dimensión de criterios de éxito, la cultura actual es jerarquizada, la deseada es clan y la requerida presenta un empate entre la cultura clan y la jerarquizada. En esta dimensión es importante que la cultura tienda hacia el tipo clan y adhocrática, y que se trabajen estrategias de desarrollo humano, trabajo en equipo y compromiso e interés por los colaboradores (figura 8, tabla 7). 
Figura 8. Resultados de cultura en la dimensión de éxito

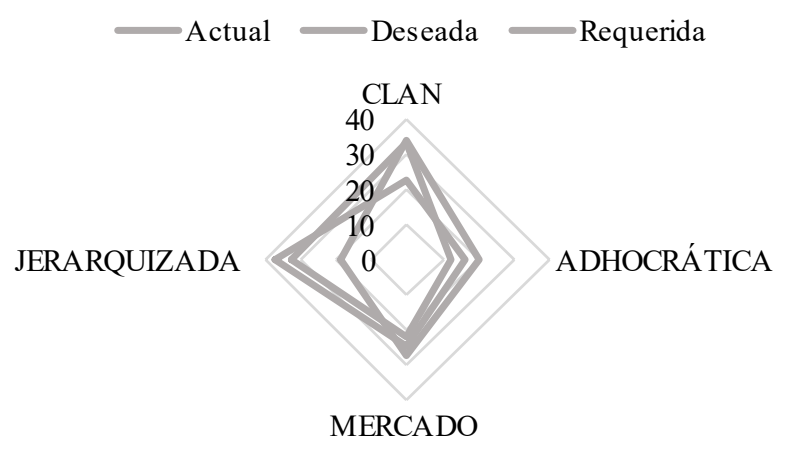

Fuente: elaboración propia.

Tabla 7. Resultados promedio de cultura en la dimensión criterios de éxito

\begin{tabular}{|l|l|l|l|}
\multicolumn{1}{|c|}{ Tipo de cultura } & \multicolumn{1}{|c|}{ Actual } & Deseada & Requerida \\
\hline Clan & 22,35 & 34 & 33 \\
\hline Adhocrática & 16,33 & 20 & 12 \\
\hline Mercado & 24,51 & 27 & 22 \\
\hline Jerarquizada & 37,55 & 19 & 33 \\
\hline
\end{tabular}

Fuente: elaboración propia.

\section{Estrategias para el cierre de brechas}

Conocer el tipo de cultura organizacional dominante de la ANH permite identificar y asociar las características deseables del estilo de liderazgo y conocer los criterios de eficacia y los modelos de gestión que la organización establece como válidos. Es necesario identificar, gestionar y lograr una adecuada interacción entre el estilo de liderazgo, los valores rectores, la gestión de los recursos humanos y los sistemas de gestión. $\mathrm{Al}$ intervenir la entidad, resulta básico identificar los actores clave, que tienen la perspectiva de la cultura organizacional global y pueden comprometerse con la implementación del cambio. De acuerdo con la 
tipología de culturas establecida por Cameron y Quinn (1999), la literatura ha destacado que la cultura clan y la adhocrática facilitan los sistemas de gestión. Por tal razón, teniendo en cuenta los resultados de la cultura actual, la deseada y la requerida, se plantean estrategias que permitan cerrar brechas y, por ende, hacer que los sistemas de gestión sean armónicos, ágiles y sencillos.

Dado que entre la cultura deseada y la requerida se identificó la cultura clan, se proponen cuatro estrategias para orientar los rasgos culturales tanto a la cultura clan como a la adhocrática. Estas estrategias se deben implementar en armonía con el proceso de implementación y mejora del Modelo Integrado de Planeación y Gestión, el cual tiene dentro de sus objetivos desarrollar una cultura organizacional sólida.

\section{Liderazgo organizacional}

Es importante articular las estrategias para fortalecer el liderazgo con las intervenciones sugeridas en la medición del clima organizacional 2019, que recomienda el fortalecimiento de las características de los líderes y de la comunicación asertiva (Compensar, 2019).

En cuanto al rol de los líderes, otros estudios afirman que aquellos que generan los más altos rendimientos han desarrollado capacidades y habilidades que les permiten tener éxito en cada uno de los cuatro cuadrantes (Cameron y Quinn, 1999). En otras palabras, los buenos líderes tienen la capacidad de identificar qué tipo de liderazgo es el apropiado para el tipo de cultura de la organización y se adaptan a este.

Se plantea una estrategia cuyo objetivo es fomentar el liderazgo a través de la identificación de responsables operativos de los diferentes procesos de la $\mathrm{ANH}$, lo que fortalecerá las habilidades gerenciales y administrativas y fomentará una cultura del reconocimiento (Covey, 1990). La estrategia contempla las siguientes etapas: 
1. Fortaler las habilidades de liderazgo.

2. Empoderar a los líderes de los procesos de la ANH.

3. Reconocimiento en los comités internos a los líderes de los procesos, en el nivel de cumplimiento de los compromisos estratégicos de la $\mathrm{ANH}$, para que desde cada proceso se tomen las decisiones oportunas y se garantice el cumplimiento de los objetivos organizacionales.

\section{Estilo de dirección}

Puesto que la comunicación es la base de cualquier relación (en este caso, la laboral) y es el vehículo fundamental para que la asimilación de la cultura organizacional por parte de los colaboradores sea óptima, se propone desarrollar una estrategia de comunicación cuyo objetivo es mantener el sentido de pertinencia. El propósito es resaltar o reconocer entre los funcionarios este buen nivel a través de campañas comunicacionales y aprovechar esta oportunidad para incrementar la apropiación, el trabajo en equipo, la participación, la gestión de las responsabilidades que exige la ANH, y la implementación de los lineamientos establecidos para el logro de los objetivos organizacionales. La estrategia se desarrolla en las siguientes etapas:

1. Ajustar el proceso de comunicación.

2. Definir espacios y medios de comunicación.

3. Dinamizar la apropiación de los valores corporativos a través de actividades lúdicas, talleres de sensibilización y campañas comunicacionales.

\section{Feedback y retroalimentación}

Para lograr los resultados de la organización en línea con la estrategia organizacional y los sistemas de gestión, se requiere que el equipo de líderes realice de manera oportuna una retroalimentación a su personal asociada con su desempeño. Esto va a permitir el alcance de los resultados 
esperados. Uno de los factores determinantes del éxito de cualquier estrategia y cultura es la capacidad de los líderes para ejercer un rol positivo y orientador, tanto de los procesos como de las personas. Son los líderes quienes en mayor medida influyen en las conductas y actitudes de sus colaboradores. La estrategia se implementa mediante las siguientes etapas:

1. Definir canales de retroalimentación y feedback.

2. Diseñar planes de desarrollo.

Gestión del conocimiento y la innovación

Para el desarrollo de esta estrategia, la ANH se debe apoyar en los lineamientos técnicos emitidos por el Departamento Administrativo de la Función Pública para la implementación de la dimensión de gestión del conocimiento y la innovación, cuyo objetivo es permitir que las entidades públicas faciliten el aprendizaje y la adaptación a las nuevas tecnologías, interconectar el conocimiento entre los servidores y dependencias, y promover buenas prácticas de gestión. Además, promueve el desarrollo de mecanismos de experimentación e innovación para el desarrollo de las soluciones eficientes en cuanto a tiempo, espacio y recursos económicos (Departamento Administrativo de la Función Pública, s. f.).

En este sentido, se propicia el desarrollo de acciones para compartir el conocimiento entre los servidores públicos, con el objetivo de garantizar su apropiación y aprovechamiento. Así mismo, se fomenta la construcción de una cultura de análisis y de retroalimentación, lo que facilita a las entidades públicas aprender de sí mismas para generar mejores productos o servicios para los ciudadanos.

Teniendo en cuenta los resultados de la ANH en la medición de las políticas de gestión y desempeño de 2018 (figura 9, tabla 8), es necesario 
seguir trabajando en el cierre de brechas y en el fortalecimiento de la dimensión de talento humano (Agencia Nacional de Hidrocarburos, 2018).

Figura 9. Resultados de las políticas de gestión y desempeño ANH, 2018

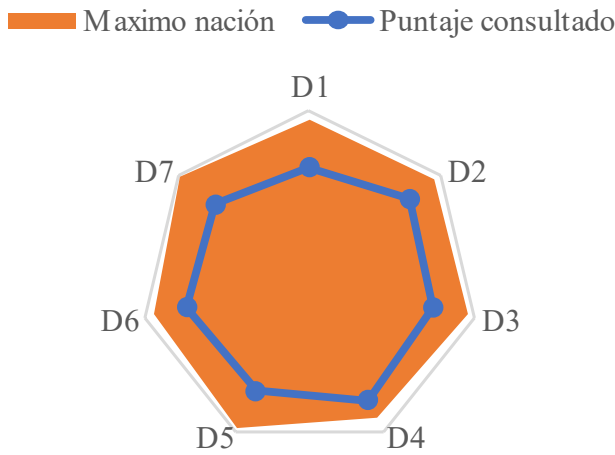

Fuente: Departamento Administrativo de la Función Pública (2019).

Tabla 8. Resultados de las políticas de gestión y desempeño ANH, 2018

\begin{tabular}{|l|l|l|}
\hline \multicolumn{1}{|c|}{ Dimensión } & Puntaje consultado & Máximo nación \\
\hline D1. Talento humano & 66,6 & 94,4 \\
\hline D2. Dirección estratégica y planeación & 76,3 & 94,9 \\
\hline D3. Gestión para resultados con valores & 75,3 & 96,2 \\
\hline D4. Evaluación de resultados & 79,6 & 90,9 \\
\hline D5. Información y comunicación & 73,9 & 98,4 \\
\hline D6. Gestión del conocimiento & 74,4 & 94,8 \\
\hline D7. Control interno & 71,1 & 97,5 \\
\hline
\end{tabular}

Fuente: Departamento Administrativo de la Función Pública (2019).

Se hace énfasis en la dimensión del talento humano porque es el eje central del sistema y el Modelo Integrado de Planeación y Gestión (MIPG) concibe al talento humano como el activo más importante con el que cuentan las entidades y, por lo tanto, como el factor crítico de éxito que les facilita la gestión y el logro de sus objetivos y resultados. El talento humano, en el marco de los valores del servicio público, contribuye con su trabajo, dedicación y esfuerzo a cumplir con la misión estatal, a garantizar 
los derechos y a responder las demandas de los ciudadanos (Departamento Administrativo de la Función Pública, s. f.). Para ello, se deben seguir estas etapas:

1. Realizar diagnóstico de la política de gestión del conocimiento y la innovación.

2. Diseñar planes para el cierre de brechas.

\section{Conclusiones}

Identificar la cultura organizacional es esencial para tomar decisiones en las organizaciones y promover los cambios necesarios para un mejor desempeño y cumplimiento de los objetivos estratégicos de la entidad. La cultura permite, asimismo, comprender las relaciones de poder en su interior, las reglas no escritas y los constructos de verdad, e identificar una serie de comportamientos considerados inicialmente como inteligibles, para dirigir las organizaciones y planear su actuación de una forma coherente con su realidad. Gestionar adecuadamente la cultura organizacional permite mejorar los sistemas de gestión y dirigirlos mediante un enfoque estratégico y cumplir con la misión y la visión organizacional.

La cultura organizacional actual de la ANH es mercado, la deseada es clan y la requerida se encuentra entre clan y mercado. Se evidencia una brecha entre la cultura actual y los tipos de cultura que favorecen la implementación y mejora de sistemas de gestión. En la cultura organizacional actual, teniendo en cuenta las diferentes dimensiones, no se identifican elementos que faciliten la implementación y el fortalecimiento de los sistemas de gestión.

Se deben desarrollar estrategias y herramientas para gestionar los cambios organizacionales, lo que implica que las personas aprendan nuevas 
experiencias, habilidades y conocimientos que permitan a los empleados entender los cambios que se pueden presentar en sus actividades laborales.

\section{Referencias}

Agencia Nacional de Hidrocarburos. (2018). Plan estratégico institucional 2018-2022.

Barahona, J. R. y Rodríguez, D. (2015). La cultura organizacional, un camino para humanizar la implementación del sistema de gestión de calidad - ISO 9001. Signos, Investigación en Sistemas de Gestión, 7(2), 19-29. https://doi.org/10.15332/s2145-1389.2015.0002.01

Cameron, K. y Quinn, R. (1999). Diagnosing and changing organizational culture based on the competing values framework. Addison-Wesley Publishing Company, Inc.

Compensar. (2019). Evaluación riesgo psicosocial.

Covey, S. (1990). Los siete hábitos de las personas altamente efectivas. Editorial Simon \& Schuster. http://www.dgsc.go.cr/dgsc/documentos/cecades/los7-habitos-de-lagente-altamente-efectiva.pdf

Departamento Administrativo de la Función Pública. (s. f.). Conozca MIPG. Consultado el 3 de diciembre de 2019. https://www.funcionpublica.gov.co/web/mipg/conozcamipg

Departamento Administrativo de la Función Pública. (2019). MIPG. Resultados medición 2018. https://www.funcionpublica.gov.co/web/mipg/resultados-2018

Hernandéz-Sampieri, R., Fernández, C. y Baptista, P. (2010). Metodología de la investigación. Mc Graw Hill Education.

Rodríguez, R. (2009). La cultura organizacional. Un potencial activo estratégico desde a perspectiva de la administración. Invenio, 12(22), 67-92. 